\title{
TERAPIA FOTODINÁMICA EN DEP AVASCULAR ASOCIADO A CORIORRETINOPATÍA SEROSA CENTRAL DE LARGA EVOLUCIÓN
}

\section{PHOTODYNAMIC THERAPY IN RETINAL PIGMENT EPITHELIUM DETACHMENT ASSOCIATED WITH LONG TERM CENTRAL SEROUS CHORIORETINOPATHY}

\author{
BORQUE E ${ }^{1}$, RUIZ O ${ }^{2}$, TORRÓN C ${ }^{2}$, FERRER E $^{2}$, ALÍAS E $^{1}$
}

\section{RESUMEN}

Objetivo: Describir un caso de coriorretinopatía serosa central asociada a un DEP crónico y el resultado de terapia fotodinámica sobre el mismo.

Caso clínico: Se presenta el caso de un hombre de 46 años con coriorretinopatía serosa central crónica de larga evolución (18 meses) con desprendimiento crónico del EPR en su ojo izquierdo que se sometió a tratamiento con terapia fotodinámica con Verteporfin (Visudyne). La AV mejoró de 0,7 a 1 , desapareciendo la metamorfopsia en un mes tras el tratamiento. El paciente permaneció asintomático durante un periodo de seguimiento de 15 meses.

Discusión: La hiperpermeabilidad coroidea es un factor primario en el desarrollo de la coriorretinopatía serosa central. La terapia fotodinámica con Verteporfin induce una reducción transitoria en el flujo sanguíneo de la coriocapilar y se puede usar como tratamiento en casos de coriorretinopatía serosa central crónica.

Palabras clave: Mácula, terapia fotodinámica, coriorretinopatía serosa central crónica, tratamiento, caso clínico.

\section{ABSTRACT}

Purpose: To present a case of long term central serous corioretinopathy associated with chronic detachment of the retinal pigment epithelium (RPE) and the effect of photodynamic therapy on it.

Case clinic: We present the case of a 46-year old man with long term chronic central serous chorioretinopathy $(18 \mathrm{mo}$.) with chronic detachment of RPE in his left eye who underwent PDT with Verteporfin (Visudyne). BCVA improved from 0.7 to 1 and metamorphopsia disappeared within one month of treatment. The patient remained asymptomatic for a follow-up period of 15 months.

Discussion: Choroidal hyperpermeability is found in central serous chorioretinopathy as a primary involvement factor. PDT with Verteporfin induces a transient reduction in choriocapilaris blood flow and can be used as treatment in cases of chronic central serous chorioretinopathy (Arch Soc Esp Oftalmol 2008; 83: 545-548).

Key words: Macula, photodynamic therapy, chronic central serous chorioretinopathy, treatment, case report.

\footnotetext{
Recibido: 9/5/07. Aceptado: 29/8/08

1 Licenciado en Medicina.

2 Doctor en Medicina.

Correspondencia:

Enrique Borque Rodríguez-Maimón

Servicio de Oftalmología

Hospital Virgen del Camino. Centro Príncipe de Viana

$\mathrm{C} /$. Irunlarrea, 3

31008 Pamplona (Navarra)

España

E-mail: eborque@gmail.com
}

Servicio de Oftalmología. Unidad de Mácula y Retina Médica. Hospital Universitario Miguel Servet. Zaragoza. España. 


\section{INTRODUCCIÓN}

Recientemente se ha comprobado la utilidad de la terapia fotodinámica (TFD) con verteporfina en el tratamiento de la coriorretinopatía serosa central crónica (CSCC) y del desprendimiento crónico seroso del epitelio pigmentario de la retina (1). Ambas entidades pueden producir un descenso de la agudeza visual. Se presenta el caso de un paciente con desprendimiento del epitelio pigmentario de la retina (DEP) seroso de larga evolución que regresó rápidamente tras el tratamiento con TFD, con mejoría de la clínica y de la agudeza visual.

\section{CASO CLÍNICO}

Paciente varón de 46 años con antecedentes de diabetes mellitus de 15 años de evolución, sin signos de retinopatía asociada, controlado en nuestro servicio anualmente por este motivo que presentó en una revisión rutinaria una imagen de DEP seroso en ojo derecho, confirmado por OCT. En la AGF no se apreciaban signos de neovascularización subrretininana subyacente (fig. 1). La AV era de 0,9 en el ojo afecto y de 1 en el ojo adelfo. La exploración oftalmoscópica del ojo izquierdo fue normal a lo largo de toda la evolución del cuadro, aunque la AGF presentaba varios puntos de hiperfluorescencia sin fuga de colorante persistentes durante todo el proceso. Durante la evolución se aprecia progreso en el tamaño del DEP mediante OCT (fig. 2) y el paciente refiere metarfopsia central.
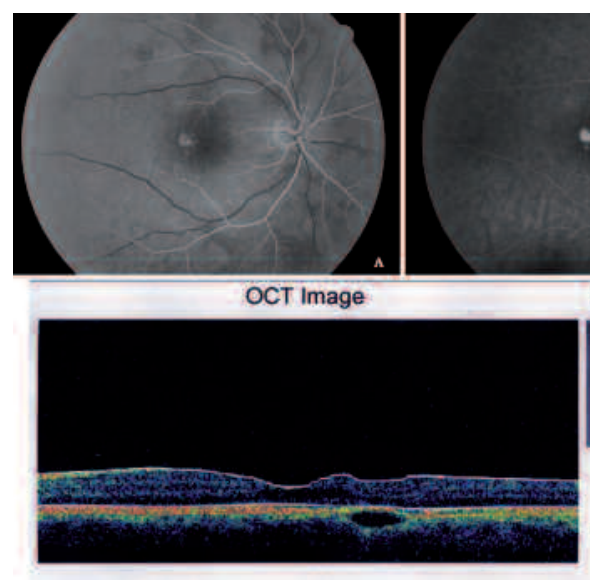

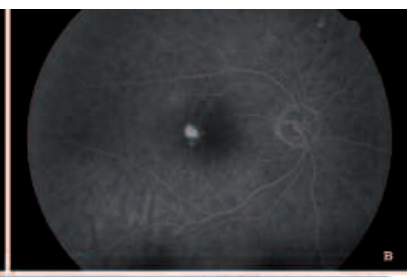

Fundus Image
Fig. 1: Angiografía fluoresceínica e imagen de OCT al diagnóstico. A: fase arterial de la AGF. B fase tardia. C: imagen tomográfica de la lesión por OCT.
A los 18 meses tras el diagnóstico el paciente presentaba una AV de 0,7 y marcada metamorfopsia con persistencia del DEP. En la AGF se apreciaba hiperfluorescencia en fase arteriovenosa con aumento de la intensidad y ligera difusión en fases tardías (fig. 3), mostrando la imagen de una alteración del EPR. Por este motivo se planteó al paciente la posibilidad de tratamiento con TFD con verteporfina como agente sensibilizante sobre el área macular afecta. El paciente aceptó el tratamiento y éste se realizó siguiendo el protocolo habitual de la degeneración macular asociada la edad (dosis de $6 \mathrm{mg} / \mathrm{m}^{2}$ de superficie corporal y spot de $2000 \mu \mathrm{m}$ ).

A las 8 semanas del tratamiento la $\mathrm{AV}$ era de 1 con corrección de la metamorfopsia. En el fondo de ojo ya no se evidenciaba ninguna bulla. La OCT mostraba una desaparición del DEP con recuperación del perfil macular normal y la AGF tan sólo mostraba alteraciones del EPR sin difusión de contraste (fig. 4). El paciente ha persistido asintomático y sin evidencia de alteración oftalmoscópica hasta ser dado de alta de nuestras consultas tras 15 meses de seguimiento.

\section{DISCUSIÓN}

La persistencia de líquido subrretiniano se asocia a una disminución de la agudeza visual en la CSCC. El tratamiento habitual de la CSC persistente en la que encontramos puntos de fuga de contraste en la AGF es la fotocoagulación con láser térmico, de esta manera podemos conseguir una resolución temporal
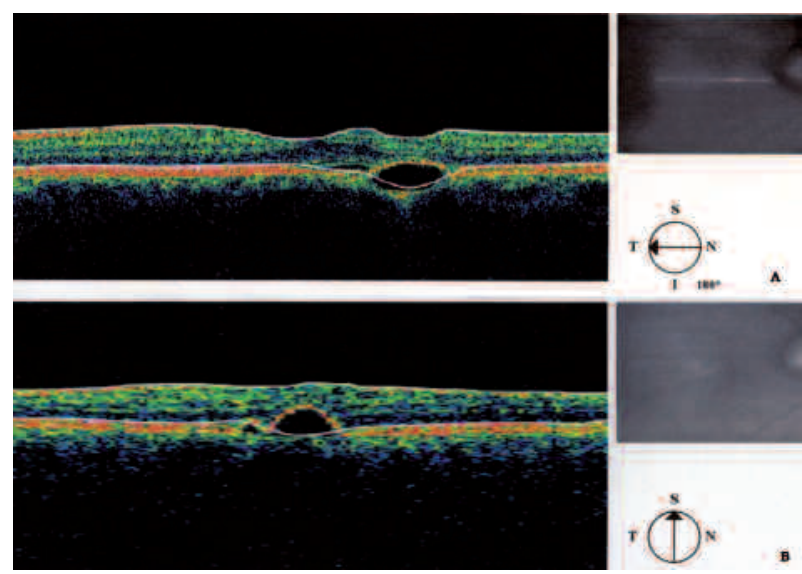

Fig. 2: Imágenes de OCT 6 (A) y 9 (B) meses tras el diagnóstico. 

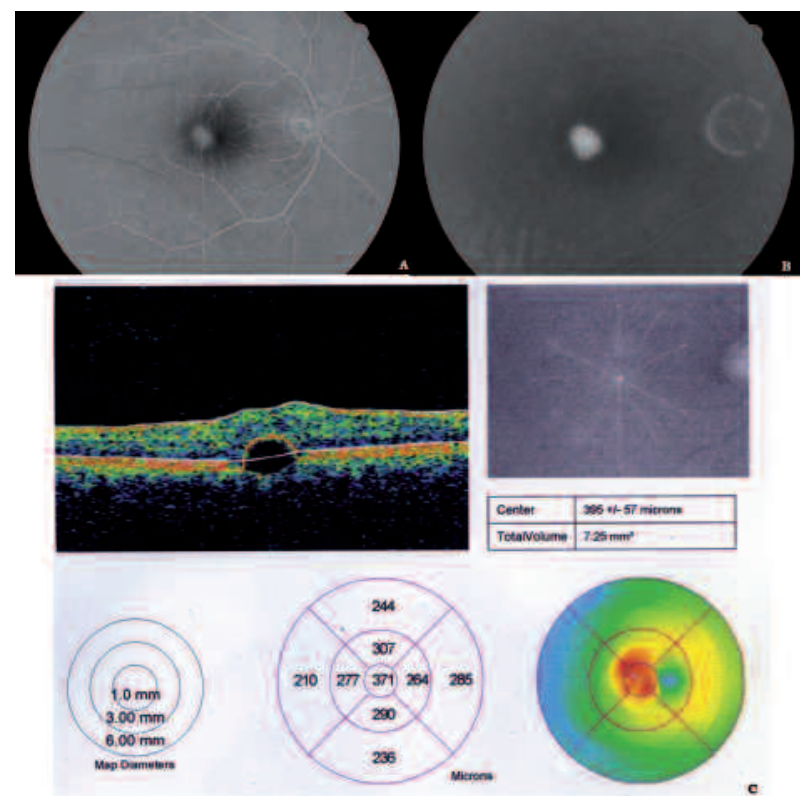

Fig. 3: AGF en fase arteriovenosa (A) y tardía (B) previa al tratamiento con TFD. Mapa macular $(C)$ de la OCT.
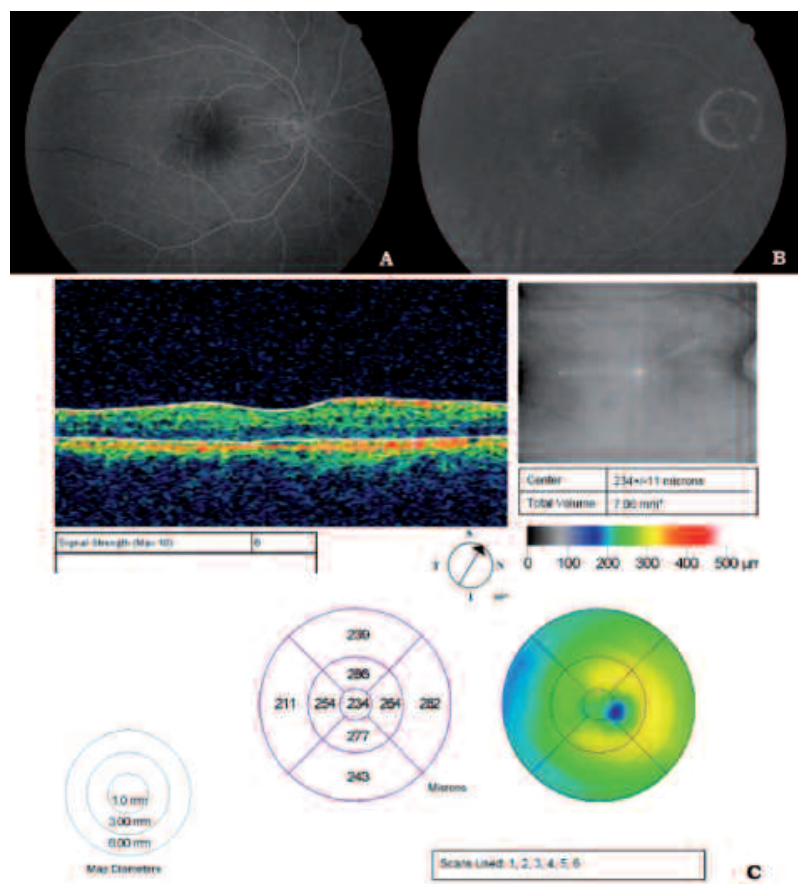

Fig. 4: AGF en fase arteriovenosa precoz (A) y en fase tardía tras el tratamiento con TFD. Mapa macular de la OCT (C). de las lesiones, aunque no se ha encontrado diferencias en cuanto a las recurrencias entre los ojos tratados con láser frente a los no tratados en nuestro medio (2). El principal problema radica en que la fotocoagulación directa con láser térmico es en ocasiones ineficaz y de resultados inciertos, especialmente en las formas crónicas de la enfermedad en las que, a menudo, el fluido emana de áreas indeterminadas de descompensación del EPR. Además el láser directo presenta diversos efectos secundarios indeseables, como la aparición de escotomas, aumento de la cicatriz atrófica con el tiempo e incluso la inducción de neovascularización coroidea.

Aunque la indicación principal de la TFD es la DMAE exudativa, últimamente se ha comprobado su utilidad para el tratamiento del DEP seroso crónico (1) no asociado a NVSR y a la CSC, tanto a sus formas crónicas $(3,4)$ como en brotes agudos $(5)$. Se ha visto en estudios con angiografía con verde indocianina (AVI) una alteración coroidea primaria en la CSC, con áreas de descompensación del EPR consecuencia de un aumento de la permeabilidad vascular coroidea. Por lo tanto, parece lógico que nos encaminemos a tratar las alteraciones coroideas desde un tratamiento más específico. La TFD puede ocluir membranas neovasculares coroideas en pacientes con DMAE. Existen evidencias de que además de producirse el efecto sobre la membrana neovascular se produce una isquemia transitoria evidenciada por AVI en la coriocapilar subyacente. Éste puede ser el efecto que reduzca la pérdida de fluido subrretiniano en la CSC, aunque los mecanismos fisiopatogénicos de esta entidad sigan siendo desconocidos.

En un estudio (4) realizado sobre 16 ojos de 13 pacientes con CSCC tratados con TFD sobre las zonas de hiperpermeabilidad coroidea evidentes en la $\mathrm{AVI}$, ningún paciente perdió $\mathrm{AV}$, presentando la mayoría de ellos una mejoría persistente. Solamente dos pacientes presentaron recurrencias en un año de seguimiento. A todos estos pacientes se les había practicado fotocoagulación directa. También se ha visto que consigue una rápida resolución de episodios de CSC aguda (5) con mejoría de la AV, especialmente en los pacientes que presentaban una mejor AV previa al tratamiento.

La mayoría de los autores coinciden en seguir los parámetros de tratamiento indicados para la oclusión de membranas coroideas neovasculares, aplicando uno o más spots no confluyentes en función de los puntos de hiperpermeabilidad vistos en la 
AGF o preferiblemente sobre las áreas de hiperpermeabilidad coroidea puestas de manifiesto en la angiografía con verde de indocianina. No se recomienda tratar dos zonas adyacentes por la posibilidad de sobredosificar la radiación en el borde de los spots. En nuestro caso no se realizó AVI, sino que se trató el área de DEP. Aún así, la mejoría de la clínica -AV y metamorfopsia- fue rápida y persistente a los 2 meses del tratamiento. Otros autores recomiendan utilizar menores dosis de radiación, bien reduciendo el tiempo de exposición (1) o disminuyendo la potencia del láser utilizado.

Se puede concluir que la TFD es un tratamiento a valorar en los pacientes con CSCC asociada a DEP sin evidencia de neovascularización subrretiniana. Es necesaria mayor evidencia sobre la patogenia de la enfermedad y sobre el papel de la TFD sobre la misma.

\section{BIBLIOGRAFÍA}

1. Olivier S, Harissi-Dagher M, Sebag M. Photodynamic therapy for chronic serous detachment of the retinal pigment epithelium in a young patient. Can J Ophthalmol 2005; 40: 214-216.

2. Torrón C, Melcon B, Ferrer E, Ruiz O, Olivan JM, Honrubia FM. Coroidopatía central serosa. Estudio a largo plazo. Arch Soc Esp Oftalmol 2000; 75: 103-108.

3. Canakis $C$, Livir-Rallatos $C$, Panayiotis Z, Livir-Rallatos $G$, Persidis E, Conway MD, et al. Ocular photodynamic therapy for serous macular detachment in the retinal pigment epitheliopathy variant of idiopathic central serous chorioretinopathy. Am J Ophthalmol 2003; 136: 750-752.

4. Cardillo Piccolino F, Eandi CM, Ventre L, Rigault de la Longrais RC, Grignolo FM. Photodynamic therapy for chronic central serous corioretinopathy. Retina 2003; 23: 752-763.

5. Ober MD, Yanuzzi LA, Do DV, Spaide RF, Bressler NM, Jampol LM, et al. Photodynamic therapy for focal retinal pigment epithelial leaks secondary to central serous chorioretinopathy. Ophthalmology 2005; 112: 2088-2094. 